\title{
Rilievo digitale delle fortificazioni di Piombino
}

\author{
Digital survey of the fortifications in Piombino
}

\author{
Stefano Bertocci ${ }^{\text {a }}$, Matteo Bigongiari ${ }^{\text {b }}$ \\ Dipartimento di Architettura - Università di Firenze, Florence, Italy \\ ${ }^{\mathrm{a}}$ stefano.bertocci@unifi.it; ${ }^{\mathrm{b}}$ matteo.bigongiari@unifi.it
}

\begin{abstract}
This paper deals with the documentation and digital survey of fortifications in the historic city of Piombino. During the Renaissance the fortified perimeter of the city was considerably enlarged, both because of the will of the Lords of Piombino, the Appiani, to build a fortified citadel, deeming the previous urban palace inadequate for the ruling family, and to improve the defenses by land with the construction of a rivellino in front of the north door and of a fortress to the east. The defenses, conquered by Valentino's troops in 1502, were redesigned by Leonardo da Vinci, as documented in the pages of Code II of Madrid, which contains drawings and calculations for the construction of the works. The present project, started in April 2018, plans to carry out an accurate digital survey of fortifications, in order to provide the basis for graphic documentation on which to base further cognitive investigations, from archaeological ones to diagnostic ones. The survey operations were carried out with today's digital technologies, laser scanners and cameras, creating reliable point clouds and three-dimensional models mapped with texture, from which to extract 2D drawings. The study of the sources, of the texts of Leonardo's scholars and accurate archaeological investigations will provide the opportunity to further analyze Leonardo's intervention on the still existing structures.
\end{abstract}

Keywords: Piombino, digital survey, fortification, Leonardo da Vinci.

\section{Cenni storici e architettonici sulle fortificazioni di Piombino}

La storia della nascita della città di Piombino è collegata alla disfatta e alla distruzione della città di Populonia (Beni, 2009) del 809 d.C. a causa delle invasioni costiere della pirateria, che in quel periodo stava saccheggiando le intere coste italiane, obbligando le popolazioni a spostarsi nelle colline dell'entroterra ${ }^{1}$. L'evoluzione della città di Piombino è stata studiata in tempi recenti (Bianchi, 2007): la storia costruttiva non presenta evidenze materiali anteriori al XII secolo, non sono state trovate tracce di strutture precedenti in nessuno scavo archeologico ${ }^{2}$, mentre il resto della costa ha visto un differente sviluppo. Il primo tracciato del castello di Piombino è stato ipotizzato in planimetria, ma le supposizioni, legate all'odierno reticolo stradale, trovano alcune lacune (Ceccarelli Lemut, et al., 2012). Piombino deve essere considerato l'insediamento più grande della zona, realizzato ex novo, frutto di un importante investimento economico dettato da Pisa (Bianchi, 2007); nel 1115 il monastero di San Giustiniano, fondato nel 1022 dalla famiglia Della Gherardesca, possedeva parte del Castello: la concessione papale del monastero potrebbe essere vista come incentivo per la fondazione di un forte centro di controllo delle coste. Allo stesso 


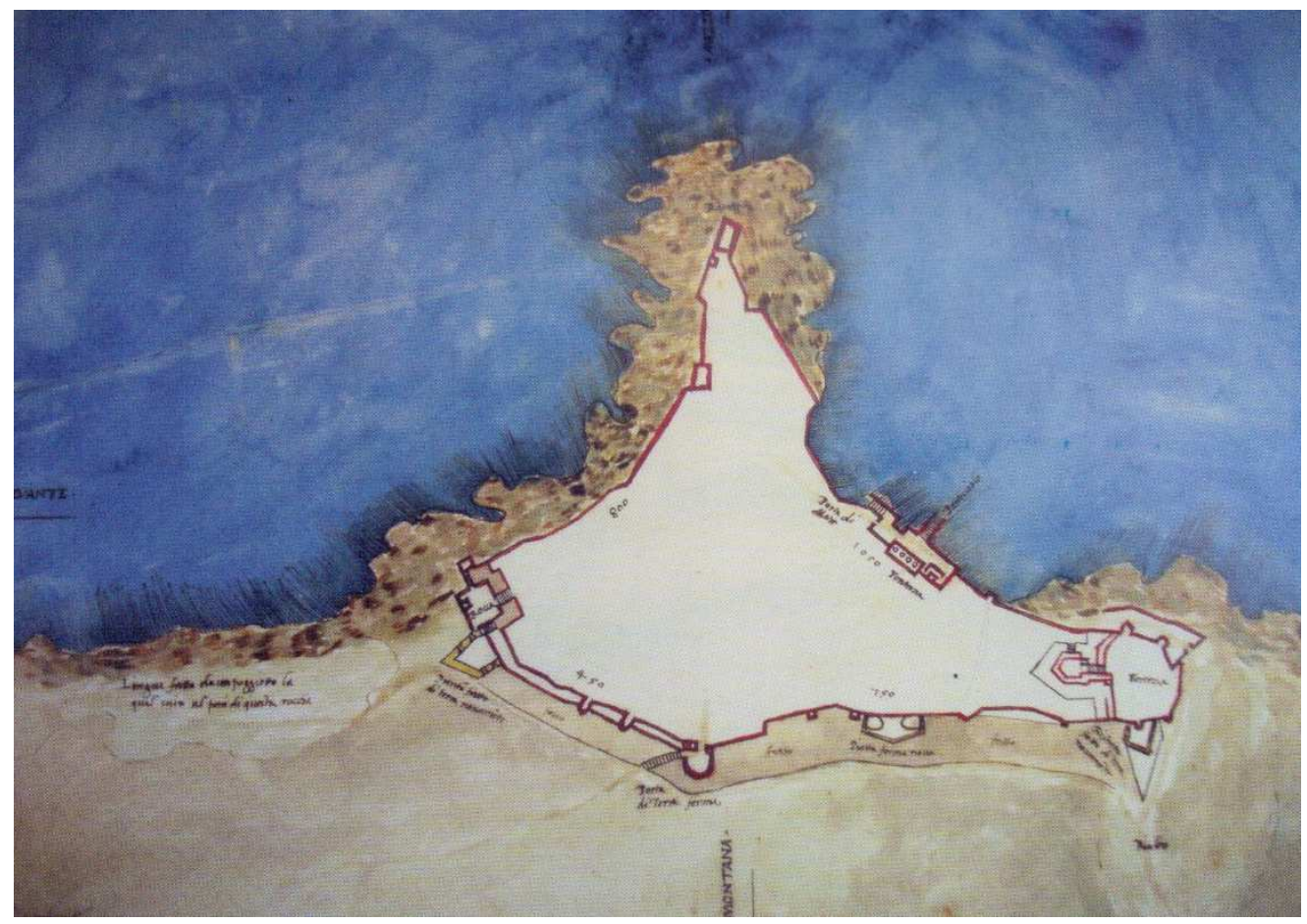

Fig. 1. Giovanni Battista Belluzzi, detto il Sanmarino, Fortificazioni di Piombino, 1522; BNF, Fondo Nazionale, II. I.280, c. 21 r.

modo un grosso intervento dell'Opera di S.Maria, con atti di compravendita del castello portarono a rafforzare la presenza pisana sul territorio. Certamente Pisa aveva una forte influenza commerciale nella zona del Tirreno fin dal X secolo, avviando una politica di incastellamento in tutta l'area maremmana. In quest'ottica Piombino divenne un punto fortemente strategico per Pisa sia per i traffici commerciali marini sia per il controllo delle città interne alla regione. L'espansione di Pisa, guidata politicamente dal suo vescovo, aveva portato all'espansione in vari centri abitati e fortificati del litorale toscano (Ceccarelli Lemut, 2005). L'importanza strategica e la fioritura economica della città all'inizio del duecento deve aver creato le basi per progettare un ampliamento del castello. Le rimanenze visibili oggi di tale operazione sul territorio e sulle planimetrie sono esigui; le uniche strutture che restano a testimonianza dell'ampliamento duecentesco sono le porte urbiche che permettevano l'accesso all'interno del nuovo limes della città: la Porta a Terra, o di San Francesco, datata al 1212, si trova oggi inserita all'interno delle strutture murarie quattrocentesche del Rivellino ampliato da Rinaldo Orsini e successivamente adeguato per resistere al tiro delle armi da fuoco (Fara, 1999); la porta all'interno del castello rinvenuta grazie ai restauri recenti della struttura (Bianchi, 2001), datata al 1235. Inoltre è visibile una porzione della cinta muraria a fianco della porta di terra; ed infine un ultimo tratto nell'angolo del porto vecchio. Il lacerto delle mura che si trova in via Leonardo da Vinci corrisponde ad un periodo successivo. Il resto delle mura è stato demolito ad inizio 900, ma rimangono a testimonianza del tracciato sia le foto storiche d'archivio, che le cartografie storiche, che i progetti depositati per distruggere le mura stesse. Solo in un periodo più tardo i documenti storici parlano dell'apertura di una Porta Nuova, nel 1282 e di una tarda Porta a Mare 1378 (Ceccarelli Lemut, 2003). Tutto faceva parte di un unico intervento urbanistico, pensato all'inizio del XIII secolo e realizzato a partire nel corso di un secolo: in tale schema rientrano le fasi di realizzazione della nuova chiesa di Sant'Antimo 
(Bianchi, 2007), e al di sotto di tale opera architettonica nello stesso periodo venne realizzata la Fonte dei Canali (1247).

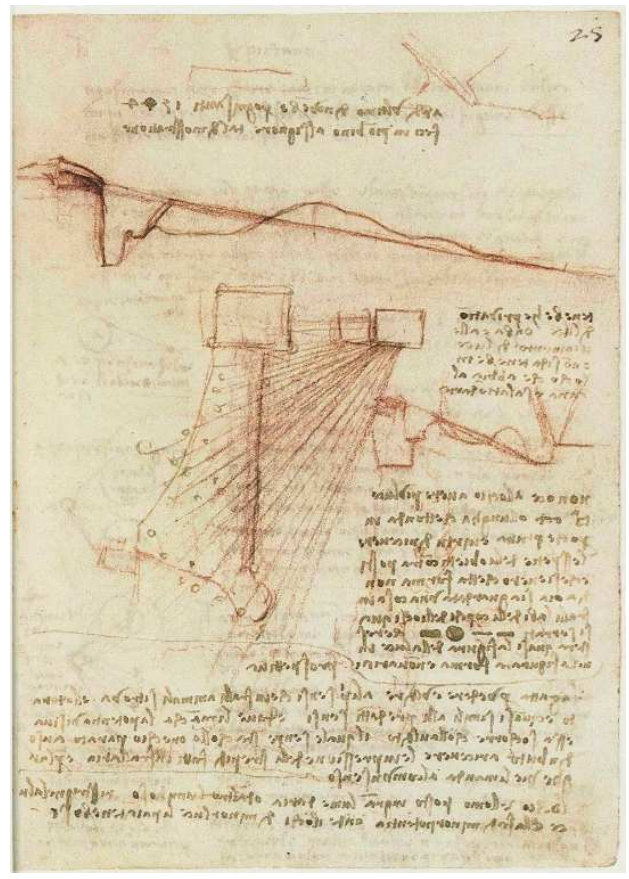

Fig. 2. Leonardo, progetto per le fortificazioni di Piombino, Madrid, Biblioteca Nazionale, Codice I, f.25r.

Piombino viene concepita a partire da due direttrici tra esse ortogonali, una nord-sud, che collega il rivellino alla Rocchetta meridionale ed una estovest, che doveva collegare il cassero al nuovo polo della Chiesa di Sant'Antimo. I documenti storici inoltre testimoniano la realizzazione di ulteriori edifici utile alla difesa militare delle coste e della città: una prima rocca nel 1330 per alloggiare i soldati pisani e un castellano, che potrebbe essere identificata con il cassero pisano. Una seconda rocca venne realizzata negli anni 70 del 1300 durante la signoria di Pierto Gambicorti a seguito di disordini interni. É stata localizzata nel luogo dove successivamente sarebbe sorta la cittadella rinascimentale [Ceccarelli Lemut 2003]; entrambe le realizzazioni furono finanziate dal governo pisano. Il castello di Piombino in periodo medievale aveva una cinta muraria che si chiudeva in se stessa lasciando all'esterno del perimetro la rocca meridionale e occidentale. Intorno al
1441 donna Paola Colonna e Angelo Orsini avevano proposto la realizzazione di palizzate lignee dalla Porta Nuova fino alla rocca di Piombino, il consiglio degli anziani approva la decisione di realizzare la difesa ma in muratura, creando un muro allo stesso modo di quello già realizzato dall'altro lato, ovvero dalla parte di villa nuova: i signori di Piombino avevano in mente di collegare la rocca occidentale all'interno del circuito fortificato della città, in modo che non rimanesse un punto di osservazione isolato. Il nuovo signore di Piombino, Jacopo III Appiani, decide che la antica residenza situata nella punta meridionale della città non è più adeguata né dal punto di vista difensivo ${ }^{3}$ né dal punto di vista del decor del Signore, capo di stato di una città moderna, prevedendo di spostarla in una cittadella da progettare ad ovest della città. La costruzione della cittadella inizia nel 1458, sull'altura dove era collocata la rocca realizzata dai Pisani, che già era stata inserita all'interno del perimetro della città di Piombino come abbiamo visto in precedenza. All'interno della cittadella viene stabilita la nuova residenza degli Appiani ${ }^{4}$. Nel 1468 l'ingrandimento della cittadella avviene tramite la costruzione di una chiesa ${ }^{5}$ e un pozzo ruotati rispetto all'asse longitudinale del palazzo, e di un corpo destinato ai servizi. Il pozzo reca iscrizioni che indicano la data 1468 e 1466 nella sottostante cisterna. Nel 1472 la cittadella assume verso la città il suo aspetto definitivo, dotando il fronte con un rivellino pentagonale ${ }^{6}$. Miguel de Corella, fedelissimo del duca Valentino, prende possesso di Piombino in qualità di luogotenente nei primi di Settembre del 1501. Vi Rimane fino all'Aprile 1502 e in quel periodo elabora e inizia a realizzare un progetto di fortificazione del fronte di terra della città. Questo progetto è rappresentato nel Codice Atlantico al foglio $115 \mathrm{v}^{7}$; il foglio è di importanza fondamentale per la comprensione del perimetro fortificato della città di Piombino alle soglie del 1500: infatti mostra il rilievo del circuito murario esistente a quella data; il fronte di terra viene riprogettato completamente di fronte alla vecchia linea difensiva, con un profilo regolare, intervallato da torri circolari e difeso da un nuovo fossato. La cittadella risulta inoltre ampliata con una 


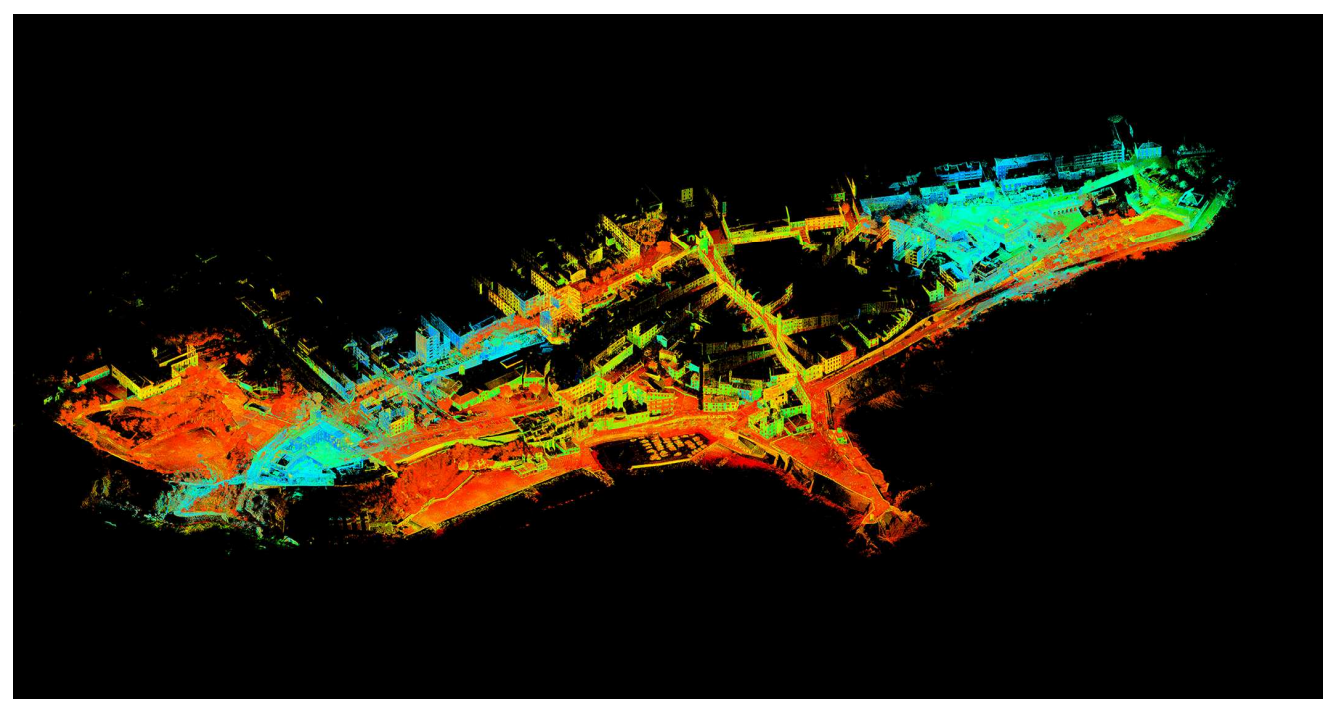

Fig. 3. Nuvola di punti tridimensionale complessiva, ottenuta attraverso acquisizioni laser scanner, della città di Piombino, che comprende tutte le sue fortificazioni.

nuovo corpo fortificato che raggiunge il poggio di Santa Maria. Gli scavi dei fossati e delle fondazioni dei muri sia del settore orientale che occidentale vengono iniziati, ma interrotti nell'agosto 1502 all'arrivo di Leonardo.

\section{Leonardo da Vinci a Piombino}

Tra ottobre e dicembre del 1504 Leonardo soggiorna a Piombino, quando è rientrato in potere al domino della città Jacopo IV Appiani. In questa occasione Leonardo propone un nuovo progetto di fortificazione criticando fin da subito apertamente il progetto ed i lavori che erano stati fatti appaltare in periodo borgiano; Dalle fonti storiche Leonardo il giorno precedente 30 di Ottobre (se si considera errata la data scritta nel taccuino: il giorno ultimo di Novembre non è infatti Ognissanti) era a Firenze, e viene inviato da Machiavelli, con l'intento di riportare armonia tra Piombino e Firenze, a Piombino per servire gli Appiani al consolidamento delle difese della città.

L'interesse per la città di Piombino da parte degli storici leonardiani è piuttosto recente e legata soprattutto al ritrovamento dei due codici madrileni conservati alla Biblioteca Nazionale di Madrid; in particolare il secondo codice è incentrato sulla esperienza di Piombino e descrive ampiamente gli schizzi di progetto e i computi metrico estimativi del costo delle opere da realizzare. Il gran numero di disegni ritrovati nel codice ha fatto riscoprire da un lato l'interesse per l'architettura militare di Leonardo da Vinci, dall'altro la concreta attività progettuale che interessava il maestro in campo di fortificazioni.

Il Manoscritto di Madrid fu in prima battuta analizzato nei disegni e trascritto (Reti, 1978). Sulla base di questa uscita iniziarono le prime considerazioni sopra i contenuti nel codice per risaltare l'operato di Leonardo da Vinci a Piombino (Heydenreich, 1974); L'approfondimento degli studi di Leonardo da Vinci a Piombino è pubblicato 25 anni dopo (Fara, 1999), analizzando più nel dettaglio i disegni del Codice II di Madrid; il merito di tale studio sta nell'indagine dell'evoluzione storica delle architetture militari di Piombino, tentando di restituire l'immagine della città che apparve agli occhi di Leonardo da Vinci quando vi giunse nelle due occasioni; incrociando le cartografie storiche, le rappresentazioni pittoriche e i documenti d'archivio del quattrocento.

Riprendendo un concetto chiave espresso da Pedretti, in occasione di un primo studio della fortezza della Verruca, e comunque universalmente condivisibile, per approfondire al meglio gli studi 


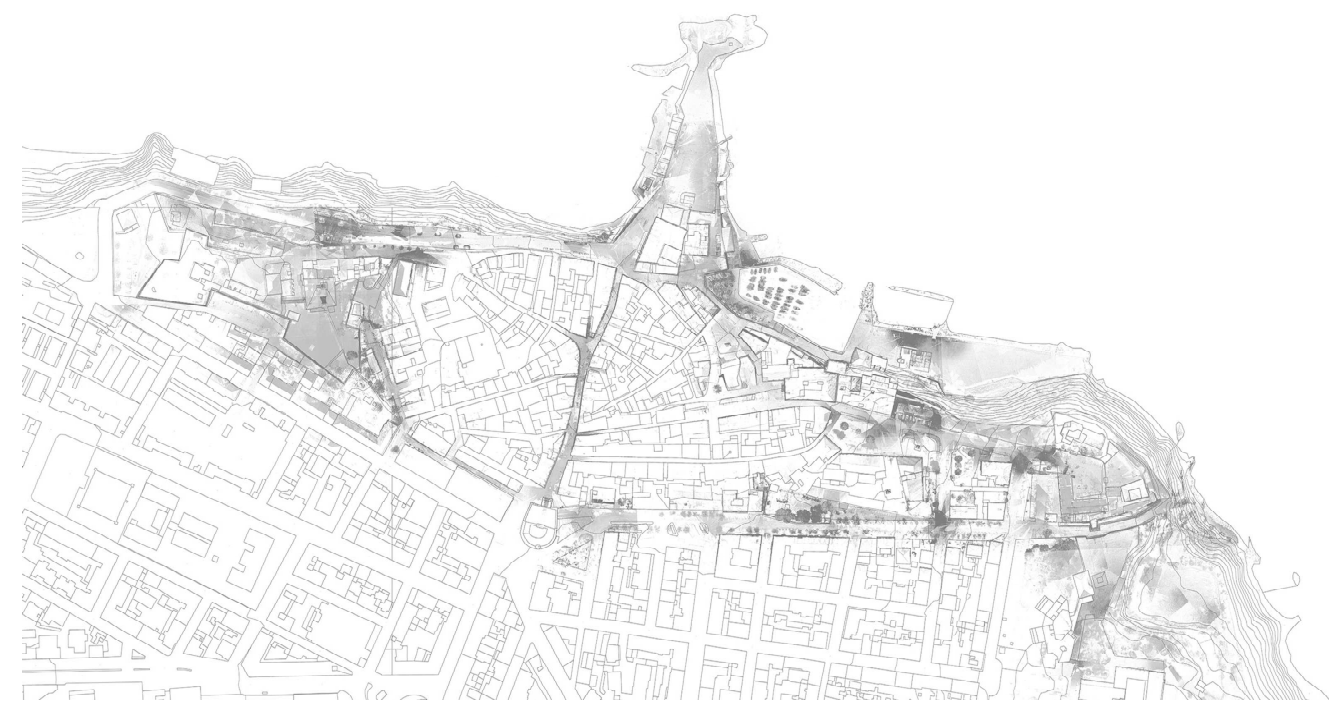

Fig. 4. Planimetria della città di Piombino, in grigio la nuvola di punti che ne descrive le architetture fortificate.

di Leonardo sulle sue architetture è necessario conoscere al meglio sia la storia che la morfologia degli edifici stessi: il rilievo architettonico, che fornisce le informazioni riguardo la forma delle architetture, è inteso in questo caso come quello strumento di conoscenza necessario all'interpretazione delle strutture e dei disegni storici; le interpretazioni dei disegni di Leonardo derivano con questa analisi da un diretto riscontro con la materia reale, concreta, con le pietre che lo stesso maestro si è dovuto trovare ad analizzare e, come vedremo, lui stesso rilevare, per conoscerle. Il rilievo architettonico, che oggi attraverso un processo continuo e esponenziale di digitalizzazione delle operazioni viene condotto attraverso strumenti sempre più sofisticati, è servito già in altre occasioni a dare importanti informazioni sull'operato di Leonardo da Vinci come architetto militare, soprattutto in quelle occasioni in cui $\mathrm{i}$ documenti storici non potevano raccontare in maniera esplicita gli accadimenti. Il rilievo dell'intero circuito fortificato di Piombino ha permesso di approfondire la conoscenza morfologica della città e delle sue architetture militari, cosa che ha conseguentemente portato al riconoscimento di numerosi ulteriori disegni e alla loro certa attribuzione alla città di Piombino.
Infine l'integrazione dei più recenti studi storici e archeologici che hanno permesso una ulteriore approfondita lettura delle fasi di realizzazione della città e delle sue architetture, hanno consentito di correggere alcune ipotesi e migliorarne la effettiva restituzione. ${ }^{8}$

\section{Il rilievo digitale}

Per comprendere al meglio le intenzioni e gli interventi pensati da Leonardo è necessario conoscere l'oggetto con cui lo stesso maestro ha interagito; e se in questo percorso di approfondimento sono stati mostrati i risultati che per lo più provengono dall'analisi documentaria, quello che ancora rimane da illustrare è ciò che le evidenze architettoniche, presenti ancora oggi sul territorio di Piombino, possono mostrare. Il rilievo geometrico si configura come una fase fondamentale all'interno del percorso che porta alla conoscenza di una architettura (Minutoli, 2017), e non vi può essere analisi completa di un edificio senza che sia stato portato a termine, quindi acquisito, elaborato e restituito graficamente, un corretto rilievo della morfologia dell'oggetto in analisi.

Per tutte queste ragioni è stato ritenuto necessario, in modo da concretizzare i dati provenienti dalla ricerca, eseguire aggiornati rilievi architettonici 


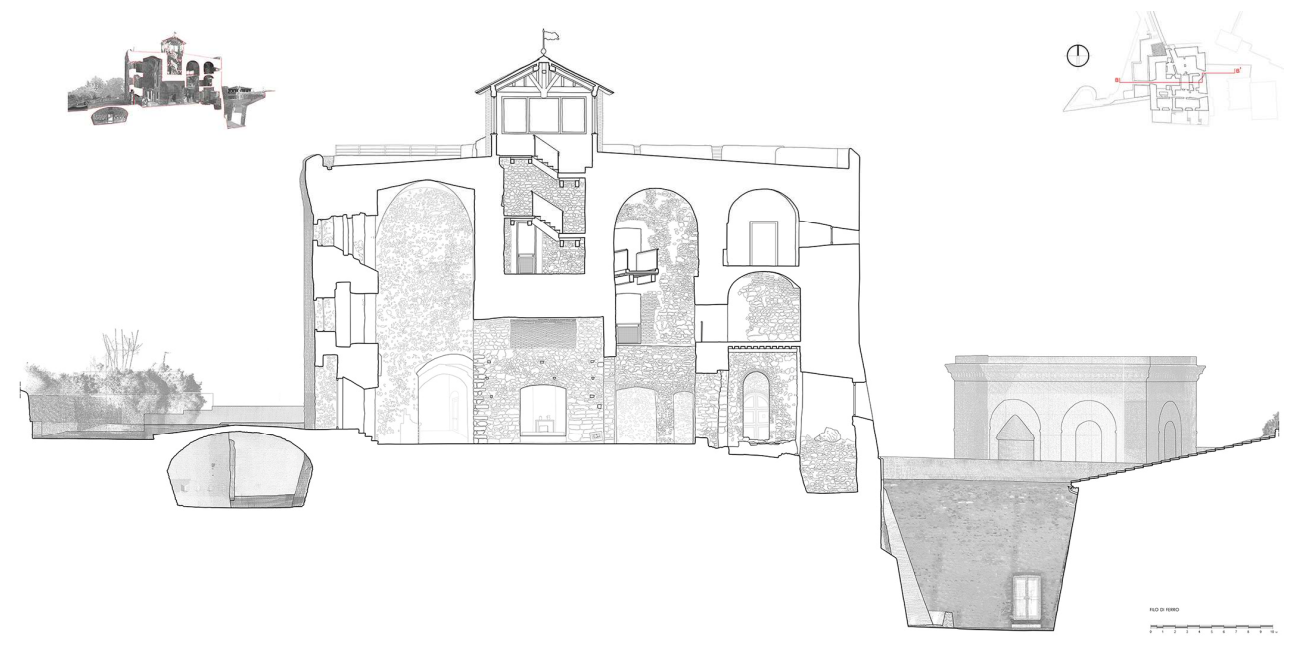

Fig. 5. Sezione a filo di ferro del Castello di Piombino, da cui sono individuabili le natiche strutture medievali del cassero pisano.

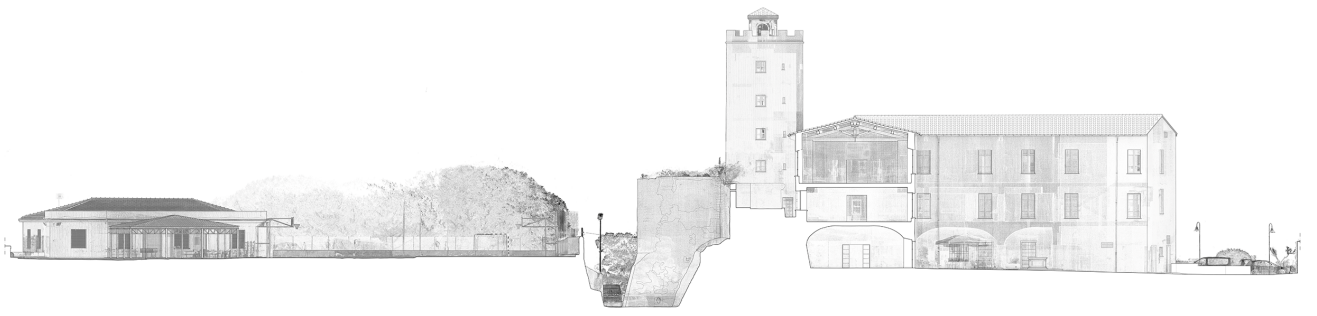

Fig. 6. Sezione a filo di ferro della Cittadella, che mostra un profilo simile a quello disegnato da Leonardo nella Fig. 2.

delle fortificazioni di Piombino, o meglio di ciò che ne resta, da utilizzare come strumento conoscitivo di base per sostenere le ipotesi che in parte sono già state descritte in precedenza sul paragrafo che tratta l'intervento di Leonardo a Piombino.

Tramite un accurata campagna di rilevamento laser scanner è stato possibile rilevare tutte le strutture architettoniche rimaste del percorso fortificato della città di Piombino; per consentire di riflettere sul rapporto in cui il sistema fortificato poneva le sue architetture è stato ritenuto fondamentale collegare in un unico modello le nuvole di punti tridimensionali ottenute delle fortificazioni: il risultato finale è stata una unica nuvola di punti che descrive la morfologia di tutto il perimetro della città quattrocentesca di Piombino, con un rilievo di dettaglio per i suoi punti focali sia delle parti esterne che intere: ovvero sono state realizzate delle nuvole di punti specifiche per il Cassero Pisano e la successiva Fortezza Medicea, il Rivellino della porta di terra, il tratto di cortina muraria adiacente alla Piattaforma progettata da Nanni Ungaro, la Cittadella Appiani. La restituzione dei rilievi è stata pensata a differenti scale, una urbanistica, in scala 1:1000 per consentire di paragonare i disegni di Leonardo su una planimetria aggiornata della città, ed una architettonica, in scala 1:50, per consentire di approfondire le analisi sulle architetture ancora oggi in piedi. Il modello proveniente dalle nuvole di punti è stato realizzato seguendo le procedure di gestione delle nuvole di punti che da molti anni sono studiate all'interno del Laboratorio di Rilievo del DiDA (Pancani, 2017). Parallelamente al rilievo 


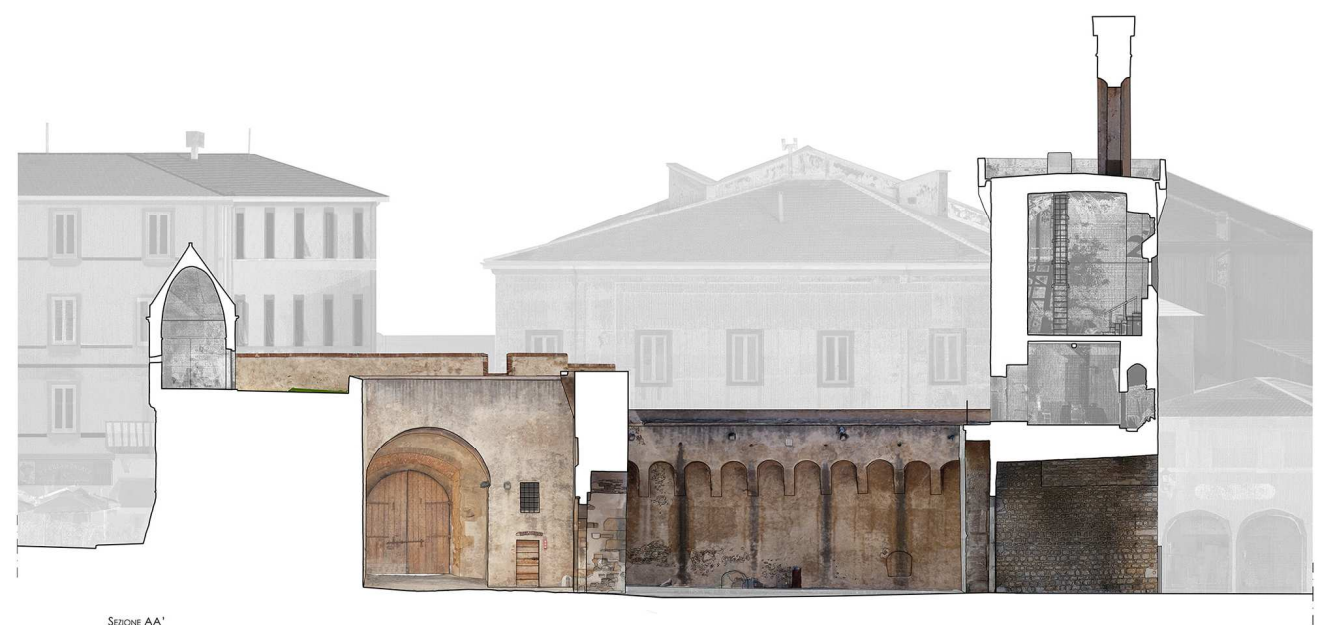

Fig. 7. Sezione con restituzione a fotopiano del Rivellino della Porta di Terra: si possono vedere le stratificazioni che dalla porta Medievale del XIII secolo giungono fino al Rivellino progettato da Rinaldo Orsini nel XV secolo.

laser scanner sono stati realizzati i modelli tridimensionali texturizzati delle architetture di Piombino attraverso l'utilizzo di camere fotografiche con processi di ricostruzione S.f.M, in modo tale da riuscire a restituire i fotopiani delle facciate su cui estendere le analisi delle stratigrafie murarie.

\section{Conclusioni}

Lo studio delle fortificazioni di Piombino attraverso sia le fonti documentarie, che le evidenze archeologiche messe in luce dalle ricerche recenti ha consentito di riassumere un chiaro modello di come la città si trovava all' arrivo di Leonardo da Vinci; il rilievo digitale delle fortificazioni ha consentito di realizzare quel corpus di documenti utili a comprendere la morfologia attuale degli edifici che compongono il sistema di fortificazioni della città di Piombino; ulteriori informazioni archeologiche potranno essere aggiunte analizzando le stratigrafie murarie delle fortificazioni rimaste in piedi, cosa che potrà fornire dati utili alla datazione delle strutture, e quindi alla attribuzione ai vari periodi storici. I rilievi realizzati saranno fondamentali per lo studio e la comparazione dei disegni di Leonardo da Vinci con lo stato attuale delle difese in modo tale da permettere di approfondire l'intervento di Leonardo stesso a Piombino.

\section{Note}

${ }^{1}$ La diocesi di Populonia venne spostata nell'interno della Val di Cornia, nell'anno 861 era “ $a d$ castrum corniae".

${ }^{2}$ Bianchi, 2007, p.387.

${ }^{3}$ Beni, 2018, p.107.

${ }^{4}$ Di questa residenza, o meglio del palazzo ottocentesco modificato per ultimo dai Baciocchi, esistono rilievi risalenti al 1855 da parte di Gaetano Rossellini; il palazzo è stato demolito nel 1959. Fara, 1999: non sembra tener conto che la residenza degli Appiani nasce proprio dove sorgeva la rocca, nelle sue ricostruzioni il palazzo rappresentato coincide con quello ottocentesco, e quindi non alla configurazione che probabilmente Leonardo trova arrivando in Piombino.

${ }^{5}$ Invece di realizzare il loggiato come una quinta scenica nella piazza della cittadella Jacopo III Appiani eresse una cappella: invece "di farci godere gl'intenzionati spettacoli, fece spettatori i Piombinesi d'una vaghissima cappella, tutta fabbricata di finissimi marmi con una soffitta di dentro da varii colori dipinta, e spesseggiata di stelle dorate" da ASPi, Franceschi Galletti, 248, Memorie, c. 43r, 1469. 
${ }^{6}$ Fara, 1999, p. 43: mostra dalle carte che anche l'anno successivo viene realizzato un ulteriore rivellino, attribuito erroneamente da Ghelardoni 1977 alla piattaforma realizzata successivamente da Nanni Ungaro.

${ }^{7}$ Fara, 1999 pp. 47-49: attribuisce non solo il progetto a Corella, ma tramite i documenti d'archivio è in grado di definire quando i vari tratti del disegno vengono appaltati per la realizzazione dapprima dei fossati.

${ }^{8}$ In questo senso gli studi sul Cassero Pisano portati avanti dalla prof. Bianchi dell'Università di Siena, le analisi dei documenti storici eseguiti dalla prof. Ceccarelli Lemut dell'Università di Pisa hanno fornito degli spunti fondamentali per la ricerca.

\section{Bibliography}

Bianchi, G. (2001). "Castello di Piombino (LI): I risultati delle ultime indagini archeologiche", Archeologia Medievale, XXVIII, pp. 185-190.

Bianchi, G. (2007). Piombino. La chiesa di Sant'Antimo sopra i canali. Ceramiche e architetture per la lettura archeologica di un abitato medievale e del suo porto, Firenze.

Beni, E. (2009). Populonia dalle origini alla fondazione di Piombino, La Bancarella editrice, Piombino.

Ceccarelli Lemut, M.L. (2003). "Piombino", in Bianchi, G., ed., Campiglia, un castello e il suo territorio, All'insegna del Giglio, Firenze, pp.55-58.

Ceccarelli Lemut, M.L. (2005). "Terre pubbliche e giurisdizione signorile nel Comitatus di Pisa (secoli XI-XIII)", in Ceccarelli Lemut, M.L., Medioevo pisano. Chiesa, famiglie e territorio, Pisa, pp. 453- 504.

Ceccarelli Lemut, M.L. (2012). Il porto di Piombino, tra storia e sviluppo, Pacini, Pisa.

Fara, A. (1999). Leonardo a Piombino e l'idea della città moderna tra Quattro e Cinquecento, Olschki, Firenze.

Heydenreich, L.H. (1974). "I progetti di Leonardo per fortificare Piombino", Almanacco italiano, LXXV, pp. 332-sgg.

Minutoli, G. (2017). Percorsi di conoscenza per la salvaguardia della città storica, DIDApress, Firenze.

Pancani, G. (2017). La Città dei Guidi: Poppi. Il costruito del centro storico, rilievi e indagini diagnostiche, Edifir, Firenze.

Pedretti, C. (2008). "Leonardo: la fortezza gustata", in Viganò, M., L'architettura militare nell'età di Leonardo: Guerre milanesi e diffusione del bastione in Italia e in Europa, Bellinzona, Casagrande. 